equipment, at which the visitor will be able to listen to the footsteps of insects, promise to enlighten the populace regarding the more spectacular aspects of scientific discovery. It is noteworthy that the site of the New York Fair, which used to be a dismal swamp outside Flushing, Long Island, has been converted into turf, suitable for planting the 10,000 trees taken to the place, by chemical treatment of the acid swamp soil.

\section{Current Sunspots}

A FAIRLY large single sunspot of area about 700 millionths of the sun's hemisphere crossed the sun's central meridian on September $25 \cdot 6$ in latitude $10^{\circ} \mathrm{S}$. This was followed by a larger group of stream type which is erossing the disk between September 21 and October 4 in latitude $12^{\circ} \mathrm{S}$., the time of central meridian passage of the group centre being September 27.7. The area of this big group on September 23 was 1,800 millionths of the sun's hemisphere. Spot groups as large as this are more often than not associated with terrestrial magnetic storms, the average time of commencement being about one day after central meridian passage. Two striking examples of the activity of this region of the sun's chromosphere were witnessed at Greenwich on September 20 and 22 with the Hale spectrohelioscope working in $H \alpha$. On September 20 at $14^{\mathrm{h}} 38^{\mathrm{m}}$ U.T., a highly eruptive prominence was observed to spring from this region then passing into view at the sun's east limb. The prominence was unusually brilliant, a photometric measure at $14^{\mathrm{h}} 41^{\mathrm{m}}$ giving a central intensity of 66 per cent (where continuous spectrum $10 \mathrm{~A}$. from the centre of $H \alpha$ at the centre of the disk $=100$ ). There were big differences of measured radial velocity amounting to $225 \mathrm{~km}$./sec. between adjacent filaments at $14^{\mathrm{h}} 54^{\mathrm{m}}$. The prominence rose from its point of origin to a height of some $4^{\prime}(175,000 \mathrm{~km}$.) within 15 minutes. On September 22, an extensive absorption marking on the disk, representing the projected image of a prominence, was observed at $8^{\mathrm{h}} 50^{\mathrm{m}}$ with a velocity of ascent exceeding $260 \mathrm{~km}$./sec. This marking, with both ascending and descending filaments, extended as seen in projection over some $150,000 \mathrm{~km}$. of the chromosphere and partly encircled the leader sunspot. Activity persisted for at least $3 \frac{1}{2}$ hours.

\section{The Night-Sky in October}

SUMmer Time ends on October 2 at $2^{\text {h }}$ U.T. The moon is full on October 9 at $9 \cdot 6^{\mathrm{h}}$ (the Hunter's moon) and new on October 23 at $8 \cdot 7^{\mathrm{h}}$. Lunar conjunctions with the planets occur as follows: On October $5^{\text {d }} 11^{\text {h }}$ with Jupiter ; October $9^{d} 12^{\text {h }}$ with Saturn; October $21^{\mathrm{d}} 4^{\mathrm{h}}$ with Mars; and on October $26^{\mathrm{d}} 0^{\mathrm{h}}$ with Venus. On October $12^{\mathrm{d}}$ at $9^{\mathrm{h}}$, Mars is in conjunction with Neptune. The planet Jupiter is the dominating bright star of the evening skies; in mid-October it souths at about $20^{\mathrm{h}}$. The various phenomena of the four inner satellites may be followed with the help of data given on pp. 618-619 of the Nautical Almanac for 1938 or in abbreviated form on p. 170 of Whitaker's Almanack. On October 16, Venus is at greatest brilliancy; but the planet sets 43 minutes after the sun. Saturn is on the southern meridian at about $23 \frac{1}{4} \mathrm{~h}$ in mid-October ; on October 8, it is in opposition, the distance from the earth being about $781 \frac{1}{4}$ million miles. Mars is a morning star rising at $3 \frac{3 \mathrm{~h}}{4}$ in the middle of the month. Two well-known variable stars are now well placed for observation during the late evenings. - Ceti, the variability of which was first recognized by Fabricius in 1596, is a long-period irregular variable. At maximum the star is usually of magnitude 3 or 4 , but is occasionally of magnitude 2 ; at minimum it sinks to magnitude 9 . The period is about $\mathbf{3 3 0}$ days. A maximum is expected near the beginning of October. The other variable, Algol ( $\beta$ Persei), is an eclipsing binary the periodic variations in brightness of which were discovered by Goodricke in $\mathbf{1 7 8 2}$ as occurring at regular intervals of 2 days 20 hours 49 minutes. There are several meteor radiants listed for October; the Orionids are seen at a maximum during October 18-20. On moonless nights, the great nebula in Andromeda, visible to the naked eye, may easily be located with the help of binoculars. The Pleiades are now well above the eastern horizon in the late evening.

\section{Announcements}

The Right Hon. the Earl of Athlone, chancellor of the University of London, will open the new buildings of Queen Mary College on October 12 at 3 p.m.

Sir Malcolm Watson will speak on "Malaria and Empire Development" at a joint meeting at 18 Northumberland Avenue, London, W.C.2, of the Planters' and Empire Social Services' Groups of the British Empire Society on October 6 at 8 p.m.

Dr. H. T. Calvert, chemical inspector at the Ministry of Health, will open a discussion on the present trend of sewage purification at a meeting of the Royal Sanitary Institute, 90 Buckingham Palace Road, London, on October 11 at 5.30 p.m.

THE Danish Institute for Human Heredity and Racial Hygiene at Copenhagen is to be incorporated in the University under the direction of Dr. Kemp.

17,330 persons have recently died of cholera in the United Provinces in the north of India during an epidemic reported to be one of the worst for many years. Bodies of victims are thrown into rivers that supply drinking water, and religious Hindus, many infected with cholera, continue to bathe in the Ganges. Mass inoculation of exposed persons and disinfection of dwellings have been carried out.

A NEw law has recently been passed in the Argentine Republic of which the principal enactments are (1) the abolition of licensed prostitution, (2) compulsory treatment for venereal disease, (3) penalties for wilful transmission of venereal disease, (4) prenuptial medical examination, which is compulsory for men and optional for women, (5) prohibition of quack advertisements for the cure of venereal diseases. 